\title{
Schwarzes Bilsenkraut (Hyoscyamus niger): Giftpflanze, Arznei- und Hexenkraut
}

\author{
Volker Unterladstetter
}

\begin{abstract}
The black bilberry is native to the Mediterranean, but also has been able to firmly establish itself in Germany. As a representative of the Solanaceae it is highly toxic and can cause hallucinations. Formerly it was added to beer. Biology, habitats, toxicity and use are presented here. A tabular comparison of the characteristics of three varieties of Hyoscyamus niger, as well as of $H$. aureus and H. albus is provided.
\end{abstract}

\section{Zusammenfassung}

Das Schwarze Bilsenkraut stammt aus dem Mittelmeergebiet, ist bei uns aber eingebürgert. Als Vertreter der Nachtschattengewächse ist es stark giftig und kann Halluzinationen verursachen. Es wurde früher auch Bier zugegeben.

Biologie, Standorte, Giftigkeit und Verwendung werden vorgestellt. Merkmale von drei Varietäten von Hyoscyamus niger, H. aureus und $H$. albus sind vergleichend als Bestimmungshilfe zusammengestellt.

\section{Einführung}

Manche Pflanzen umgibt Dunkles. Das gilt nicht zuletzt für einige Vertreter der Nachtschattengewächse (Solanaceae), aus deren Reihen die Tollkirsche (Atropa bella-donna) als eine der giftigsten Pflanzen Mitteleuropas bekannt ist. Ein weiteres von Mythen umranktes Gewächs aus dieser Familie ist das Schwarze Bilsenkraut (Hyoscyamus niger), eine Gewächs stickstoffreicher, von Menschen beeinflusster Standorte. Aufgrund des Gehaltes an verschiedenen Alkaloiden sind alle Teile der Pflanze giftig, der Genuss ruft halluzinogene Zustände hervor „und verleiht Hexen Flügel“.

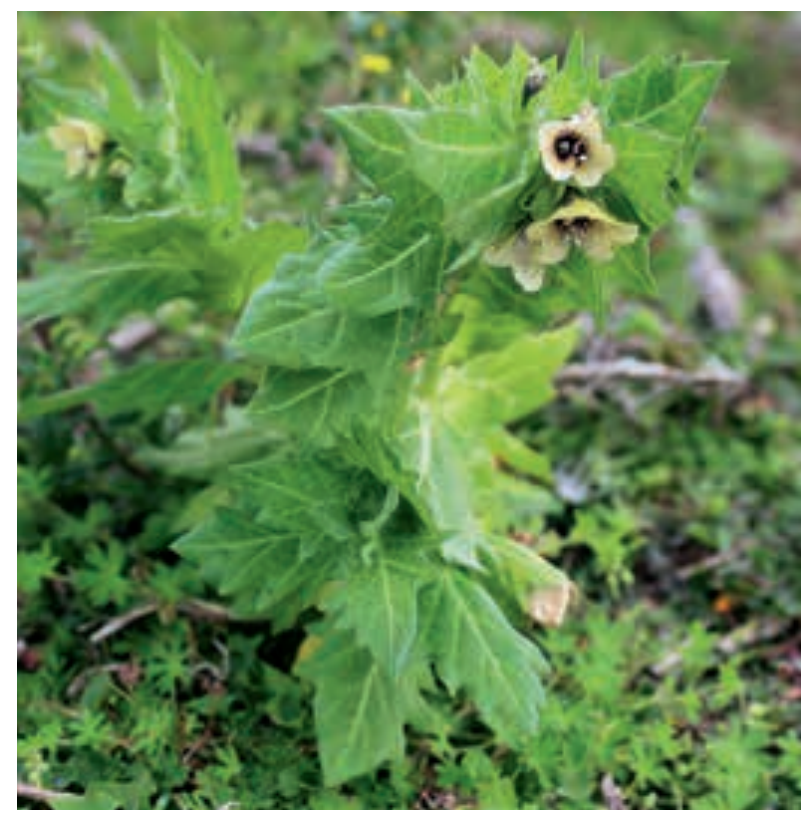

Abb. 1: Hyoscyamus niger.

\section{Morphologische Merkmale}

Das Schwarze Bilsenkraut ist eine ein- oder zweijährige Pflanzenart aus der Familie der Nachtschattengewächse (Solanaceae), die einzige in Deutschland dauerhaft vorkommende Art aus der Gattung der Bilsenkräuter (Hyoscyamus). Es erreicht eine Größe von $20-80 \mathrm{~cm}$ und beginnt mit der Blütenbildung als Langtagpflanze erst, wenn die Tageslänge eine Dauer von 11 Stunden überschreitet (Düll \& KutZelnigg 2016). In älteren Arbeiten wird der Blühbeginn meist mit Juli angegeben (z. B. Laven \& Thyssen 1959), in neueren Werken finden sich dagegen Angaben zu

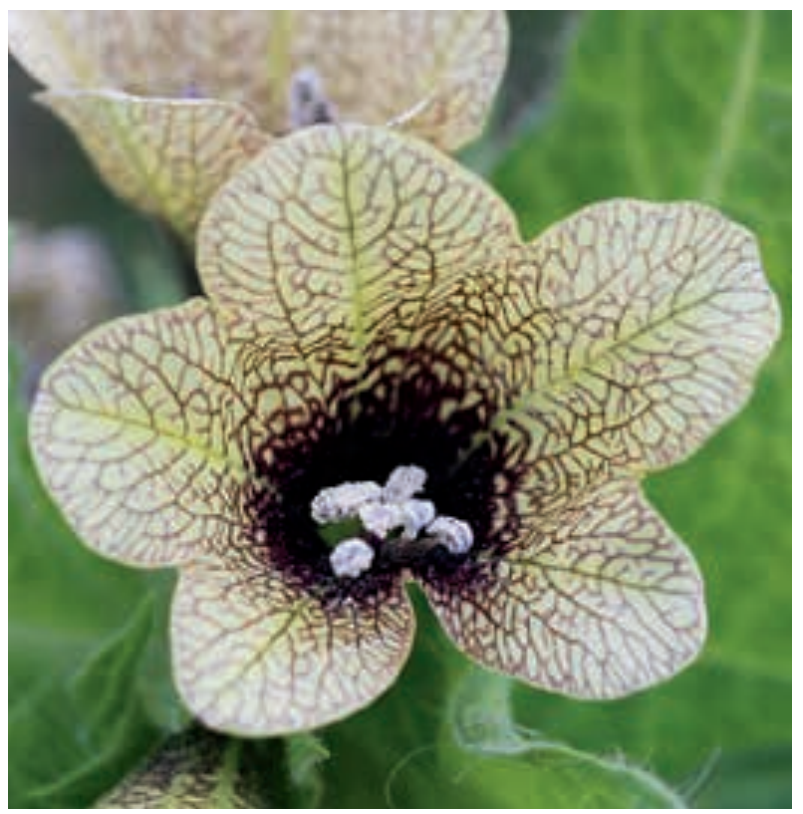

Abb. 2: Blüte von Hyoscyamus niger. 


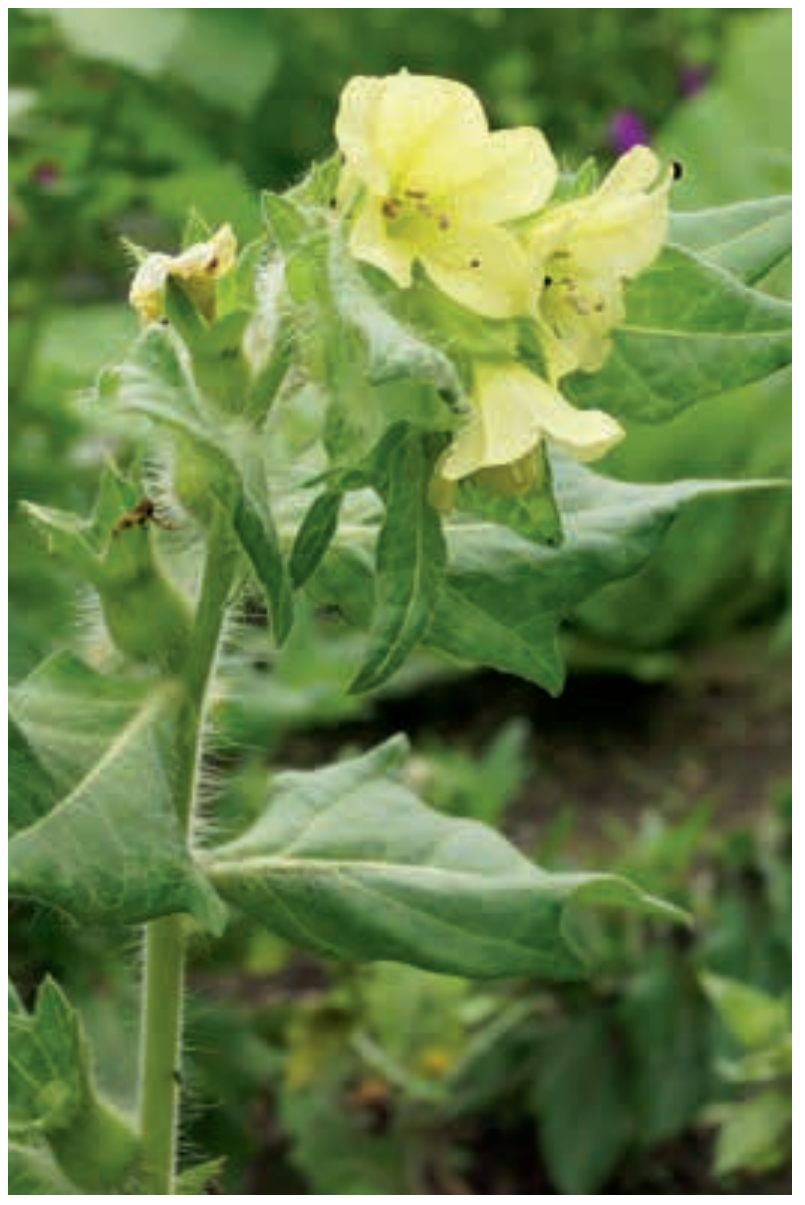

Abb. 3: Hyoscyamus niger var. pallidus mit hellgelben Blüten ohne schwarze Zeichnung.

einem früheren Blühbeginn im Juni. Ein im Jahr 2017 in einem Kölner Stadtpark auf gestörtem Boden über einer alten Deponiefläche vorgefundenes Exemplar stand hingegen schon Mitte Mai in Blüte. In einer Arbeit von Schläppi (2011) wurde nachgewiesen, dass es mehrere unterschiedliche Blühsippen gibt, die sich sowohl in ihrer Entwicklung (einjährig, zweijährig) als auch in ihrer Blüteninduktion unterscheiden (unterschiedliche Empfänglichkeit für Vernalisation und Einfluss der Fotoperiode). Dabei konnte bei Kreuzungsexperimenten gezeigt werden, dass für die zweijährige Entwicklung ein einziges dominantes Gen verantwortlich ist.

Der Habitus der Pflanze ist je nach Nährstoffverfügbarkeit und Lichtexposition aufrecht und breit verzweigt bis wenig verzweigt oder unverzweigt. Der Stängel ist stumpfkantig, klebrig-drüsig und auffällig weiß und dicht behaart, ebenso der Kelch und die Blattstiele. Die Blätter werden bis zu $20 \mathrm{~cm}$ lang, sind wechselständig, eiförmig und buchtig gezähnt, gelappt oder fiederteilig, zum Teil auch ganzrandig. Während die unteren Stängelblätter gestielt sind, sitzen die oberen Blätter dem Stängel an und umfassen ihn. Nach Angaben einiger Autoren ist das Kraut von unangenehm betäubendem Geruch, der auf das Alkaloid Hyoscyamin zurückgeführt wird und vor Tierfraß schützen soll (Beckhaus 1893, Hegi 1975, Düll \& Kutzelnigg 2016).

Die Blüten des Bilsenkrautes stehen im Blütenstand einseitswendig, einzeln in den Achseln großer Tragblätter. Sie sind mehr oder weniger ungestielt und haben einen glockenförmigen Kelch mit stechenden Zipfeln. Der Kelch ist während der Blütezeit engglockig, vergrößert sich aber zur Fruchtreife und schließt die Frucht ein (Aichele \& Schwegeler 2000). Die Kelchblätter aller Hyoscyamus-Arten sind miteinander zu einer ausdauerngen Röhre verwachsen, die im Bereich der Leitbündel vermehrte Sklerenchymbildung aufweist (FuKAREK 2000). Zum Zeitpunkt der Fruchtreife ist der Kelch bereits vollständig eingetrocknet und umschließt die Frucht.

Die Kronblätter sind zu einer Röhre verwachsen, die sich nach außen hin radiärsymmetrisch in fünf Kronblattzipfel zu öffnen scheint. Die Blüten sind jedoch schwach zweiseitig symmetrisch. Ihre Grundfarbe ist von einem matten, schmutzigen Gelb, der Schlund ist bei der Typusvarietät $H$. niger var. niger durch die Einlagerung von Anthocyanen dunkelviolett gefärbt. Die gleiche Färbung weisen auch die Saftmale auf, die vom Schlund nach außen in die Zipfel verlaufen. Vielfach verzweigt erscheint diese Netzaderung bei näherer Betrachtung wie eine filigrane Strichzeichnung.

Die Blüte beginnt bei $H$. niger mit einer weiblichen Phase, in der der Griffel mit der Narbe weit aus der Kronröhre herausragt. Zu diesem Zeitpunkt befinden sich die Antheren noch oberhalb des Griffels und sind geschlossen. In der nachfolgenden männlichen Phase schieben sich die Staubgefäße mit den Antheren an die Narbe heran, so dass eine Selbstbestäubung der Blüte stattfinden kann (Möвıus 1931, Hegi 1975). Nachdem sich 


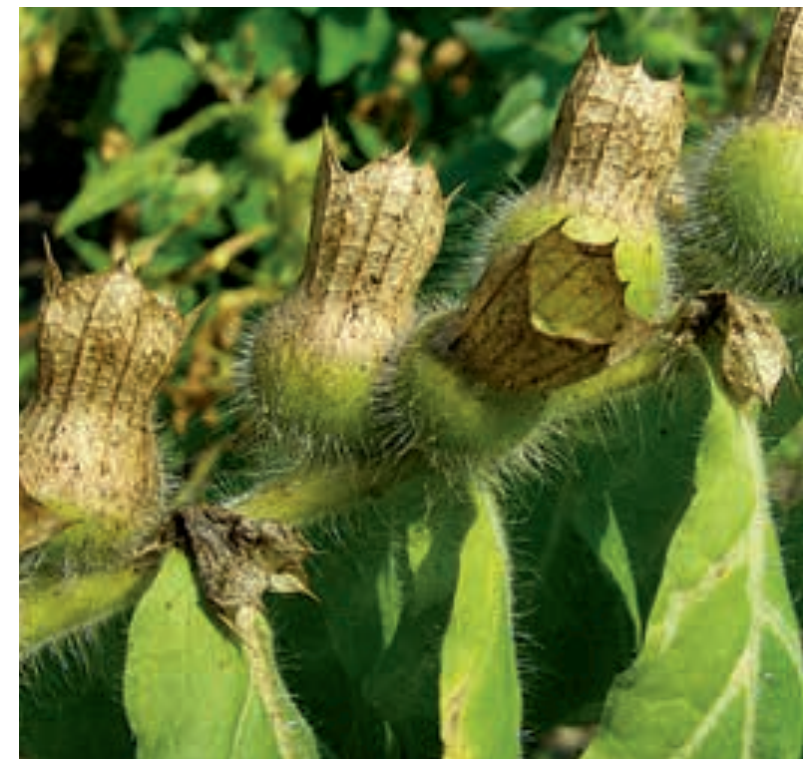

Abb. 4: Ausschnitt aus dem Fruchtstand von Hyoscyamus niger.

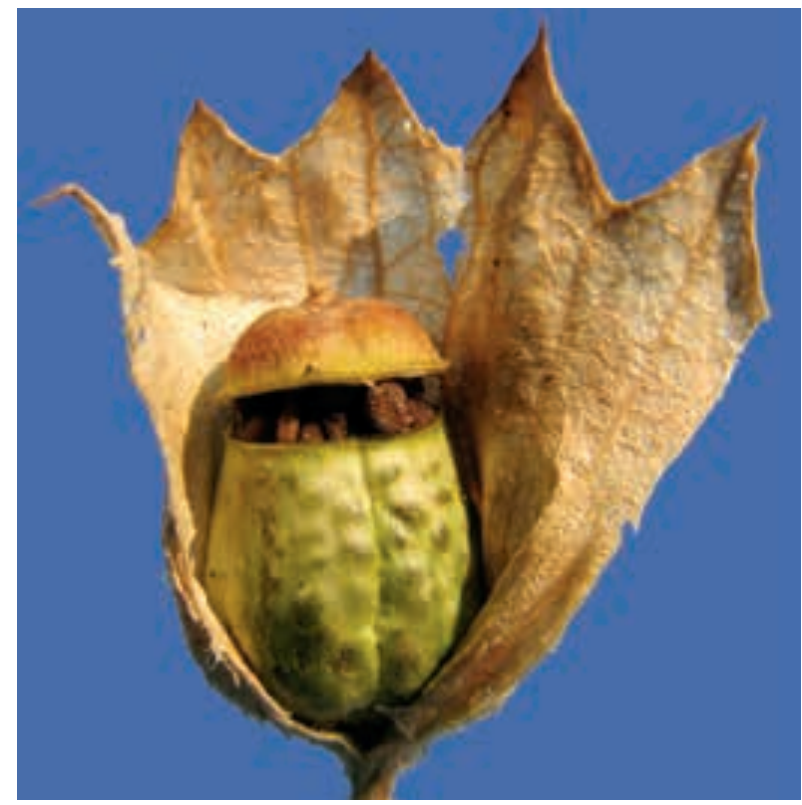

Abb. 5: Sich öffnende Kapsel von Hyoscyamus niger.

die Antheren geöffnet und entleert haben, biegen sich die Filamente zurück.

Wenn die Blühphase mit der (Selbst-)Befruchtung der Blüte geendet hat, bildet sich eine Kapselfrucht. Anders als bei den meisten Kapseln reißt diese bei der Reife nicht entlang der Verwachsungsnähte der Fruchtblätter auf, sondern es trennt sich der obere Teil ab und bildet eine sogenannte Deckelkapsel. In einer Kapsel befinden sich etwa 200 (bis 500) nierenförmige Samen, die $1 \mathrm{~mm}$ lang und etwa $1,3 \mathrm{~mm}$ breit sind (HegI
1975, Philip pi 1996). Die Samen von H. niger sind äußerst langlebig und sollen ihre Keimfähigkeit über 600 Jahre behalten, weshalb die Pflanze auch an unerwarteten Stellen aus gestörten Böden aufkeimen kann (Düll \& Kutzelnigg 2016).

\section{Etymologie}

Bereits die Schreibung der Gattung entlarvt Hyoscyamus als griechischen Namen (der später latinisiert wurde): gr. hys/hyos bedeutet Schwein, gr. kyamos ist die Bohne, beides zusammen ergibt hyoskyamos, die Saubohne. Genaust (2005) spekuliert über eine mögliche Analogie zwischen den Samen der Saubohne (Vicia faba) und der bauchigen Deckelkapsel der Bilsenkräuter.

Der Artbeiname niger bezieht sich auf die Blüte. Im Gegensatz zur verwandten Art Hyoscyamus albus sind die Adern (Saftmale) der Kronblätter bei $H$. niger im gleichen Dunkel-(schwarz)-violett gefärbt wie der Schlund.

Die Bedeutung des deutschen Namens bleibt weitestgehend unklar. Düll \& Kutzelnigg (2016) schlagen eine Verwandtschaft des heute undurchsichtigen ersten Bestandteils Bilsen zu althochdeutsch pilisa $=$ tödlich vor und heben damit die tödliche Giftigkeit bei einer oralen Anwendung der Pflanze hervor. Kluge \& Seebold (2002) führen den Namen auf bilisa zurück. Dazu werden verwandte Benennungen in weiteren Sprachen gestellt, zum Beispiel altprovenzalisch belsa, altenglisch belene/be(o)lone oder russisch belená. Eine Möglichkeit, hier eine gemeinsame Urbedeutung zu rekonstruieren, besteht mit Rückgriff auf eine rekonstruierte indogermanische Verbalwurzel ${ }^{*} b^{b} l e n d^{b}$-, die mit verwirren angegeben wird (vgl. auch deutsch blind). Eine solche Bedeutung könnte tatsächlich zugrunde gelegen haben, wenn man sich vor Augen führt, dass die Bilsenkräuter seit der Antike als halluzinogenes Mittel eine bedeutende Rolle gespielt haben.

Geradezu unüberschaubar gestaltet sich die Landschaft der Regionalnamen im deutschen Volksmund. Marzell (1972) listet eine Fülle von Benennungen auf. So finden sich mundartlich neben Bilsenkraut viele weitere Varianten, 


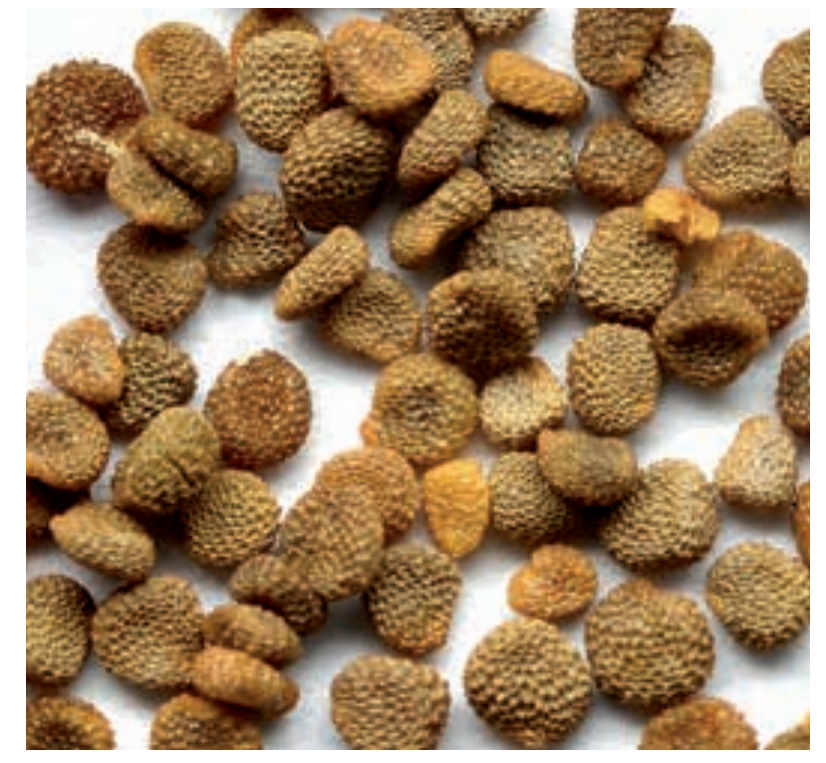

Abb. 6: Samen des Schwarzen Bilsenkrautes.

beispielsweise Pilsenkraut, Bülsenkrût, Belzkraut, Birmeskraut, Billenkrût, Bimselkraut, Binsenkraut und dergleichen mehr. Andere Benennungsmuster beziehen sich auf die Wirkung der Pflanze auf den Menschen (Tolle Bülsen, Dülledill, Tollkraut - vgl. auch engl. insane-root -, Raasewurz, Dummkraut, Schlaffkraut, Zankteufel) oder auf die generellen Auswirkungen ihres Giftes (Gänsegift, Hühnertodt, Todtenkräutel).

Namen wie Hühnerlockkraut bezeugen alte Praktiken, die ganz offensichtlich nicht der heutigen Tierwohlethik entsprachen. Nach einer Beschreibung in Hieronymus Bocks Kräuterbuch von 1539 werden Hühner durch den Rauch von Bilsenkraut dermaßen betäubt, dass sie von ihrer Stange fallen (Marzell 1972: 930f.). Auch den Schweinen bekam die Bilse nicht sonderlich gut, wie aus einigen Benennungen (Saugift) und der Literatur zu entnehmen ist. Schweine, die von der Wurzel der Pflanze gefressen haben, drückten ihre Bäuche an den Boden und schleppten sich kriechend vorwärts (MARZELL 1972).

\section{Vergesellschaftung und Ökologie}

Durch ihre Liebe zu nährstoffreichen Störstellen ist Hyoscyamus niger der alten, heute beinahe verschwundenen mitteleuropäischen Dorfflora zuzuordnen. Zusammen mit anderen Nährstoffzeigern wie Eselsdistel (Onopordum acanthium) und Hundszunge (Cynoglossum officinale) gilt das Bil- senkraut nach Ротт (1995) als Kennart der Eselsdistel-Flur (Onopordetum acanthii), die stellnweise seltener wird und z. B. im Westfälischen Gebiet heute nicht mehr vorkommen dürfte (LIENENBECKER \& RAABE 1993). Eselsdistel-Fluren gehören zu den thermophilen Distel-Gesellschaften, die im Verband Onopordion innerhalb der Klasse der Mehrjährigen ruderalen Staudenfluren und Säume (Artemisietea vulgaris) zusammengefasst werden. Pотт führt als Assoziationscharakterarten neben der Eselsdistel die Großblütige Königskerze (Verbascum densiflorum) und die Windblumen-Königskerze (Verbascum phlomoides) an.

Hyoscyamus niger wächst an trockenen, wärmebegünstigten Standorten an licht- und basenreichen Stellen: am Fuße von Mauern, auf Schuttplätzen, auf Ruderalflächen in Siedlungsnähe und generell an Störstellen mit offenem Boden. Ihre frühere Nutzung als Heil- und Zauberpflanze wird besonders durch Fundorte in der Nähe von alten Burgen und Siedlungsplätzen deutlich. Die Art ist heute vielerorts (so auch in Nordrhein-Westfalen) selten geworden und taucht oft nur unbeständig auf (vgl. z. B. Haeupler \& al. 2003, Gorissen 2015). In der Roten Liste von NRW beispielsweise wird sie entsprechend als stark gefährdet geführt (RAABE et al. 2011). Mit Hinweis auf ursprünglich fehlende Primärstandorte wird allgemein vermutet, dass das Schwarze Bilsenkraut ein Archäophyt ist (Düll \& KutzelnigG 2016). Allerdings muss die Einbürgerung schon in vorgeschichtlicher Zeit erfolgt sein, denn bereits von den alten Germanen wird berichtet, dass sie Bilsenkraut als Pfeilgift benutzt hätten (НАЕ RкӧтTER \& HaerkötTer 1987).

\section{Historische Verwendung}

Das Schwarze Bilsenkraut kann trotz seiner ungünstigen Allgemeinwirkung auf den Menschen auf eine beeindruckende Nutzungsgeschichte zurückblicken. Bereits in der Antike waren die Bilsenkräuter den Gelehrten wohlbekannt. Der griechische Arzt Dioskurides beschreibt in seiner Materia medica aus dem 1. Jh. n. Chr. die drei damals bekannten Arten der antiken Welt. Von diesen brächten jedoch Hyoscyamus niger und $H$. aureus Wahnsinn und Lethargie über den $\mathrm{Pa}-$ 


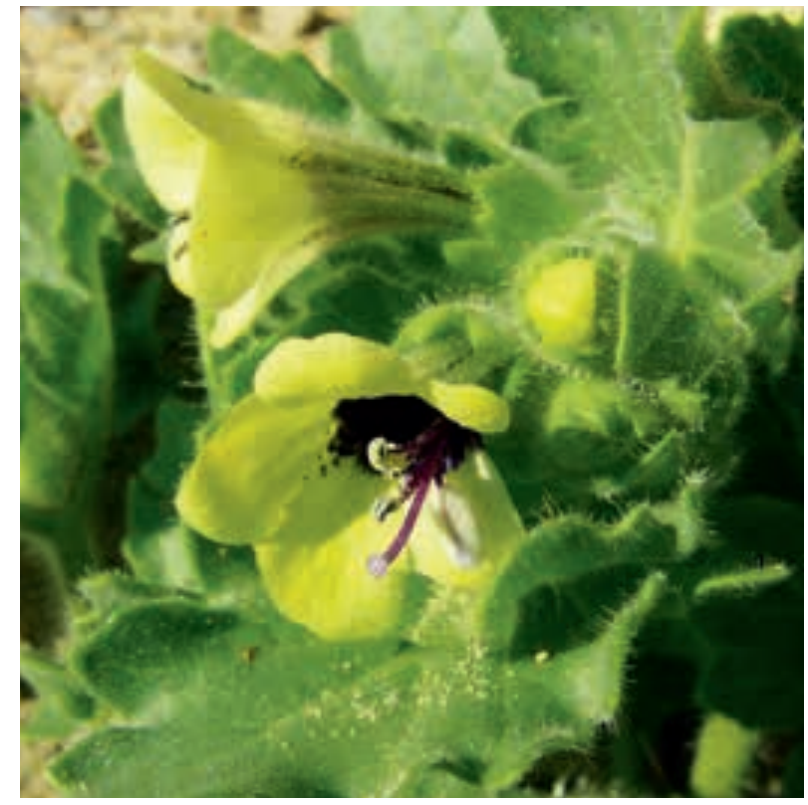

Abb. 7: Hyoscyamus albus, auf Mallorca.

tienten und seien damit als Arzneipflanzen ungeeignet. Das Schwarze Bilsenkraut wurde somit bereits in der Antike als gesundheitsgefährdend erkannt. An anderer Stelle wirkte ein gewisser Wahnsinn hingegen durchaus berufsfördernd: So wird berichtet, dass die Priesterinnen des Orakels von Delphi ihre weitsichtigen, wenn auch meist wenig eindeutigen Weissagungen unter dem Einfluss von verräuchertem Bilsenkraut tätigten (vermutlich des Weißen Bilsenkrauts, H. albus, Prentner 2004).

Der Popularität des Krautes in Mitteleuropa hat der antike Verriss keinen Abbruch getan, auch wenn die Giftigkeit den mittelalterlichen Autoren ebenfalls wohlbekannt war. Wahlweise wurde Schwarzes Bilsenkraut als Heilpflanze eingesetzt (zum Beispiel als Mittel gegen Schmerzen aller Art, gegen Lungenleiden oder bei geschwollenen Hoden), als Giftpflanze, Aphrodisiakum, Narkotikum bei Operationen oder als Zauberpflanze. Obwohl schaurige Geschichten über Giftmorde kursierten, blieben tödliche Vergiftungen aufgrund der geringen Gesamtgehalte der beteiligten Alkaloide (vor allem 1-Hyoscyamin, Atropin und Scopolamin) wohl eher die Ausnahme (НаerkötTER \& HAERKÖtTER 1987). Eine besondere Rolle spielte bei der Verwendung das Scopolamin, das zu Halluzinationen führt und darum auch in Hexensalben Anwendung fand. Duve \& Völker (2002)

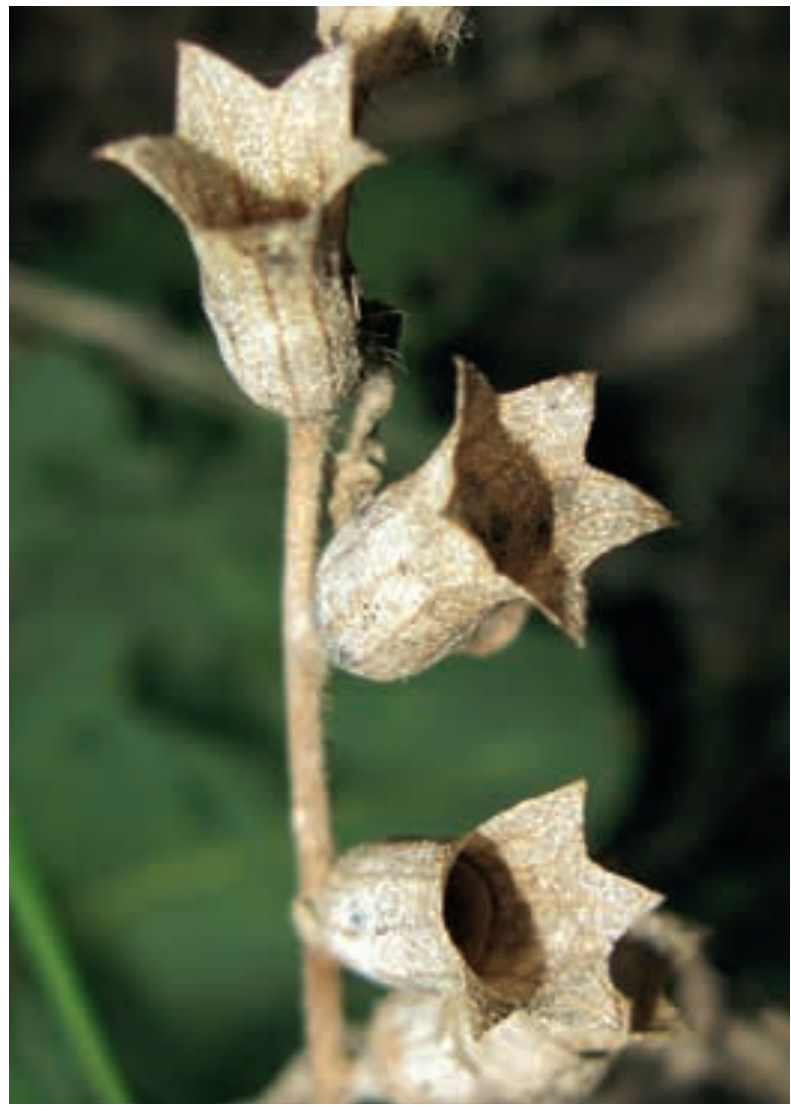

Abb. 8: Ausschnitt aus dem Fruchtstand von Hyoscyamus albus.

bemerken dazu, dass die eingebildeten Flugabenteuer der Hexen möglicherweise der halluzinogenen Wirkung des Bilsenkrauts zuzuschreiben seien (oder aber der Fantasie der Inquisitoren).

$\mathrm{Zu}$ den körperlichen Auswirkungen von Bilsenkrautgenuss zählen unter anderem Hautrötungen, Pupillenerweiterung, Mundtrockenheit, Herzrhythmusstörungen, verminderte Sinneswahrnehmung, Erregung mit Tobsuchtsanfällen sowie ein tiefer Wunsch nach Schlaf (Наerкötter \& Haerkötter 1987, Prentner 2004, Rotн et al. 2012). Andere Autoren verweisen auf die unfreiwillige Komik der Betroffenen, die von Fröhlichkeit mit lächerlichen Stellungen und Gebärden (Sснміdт 1840) heimgesucht würden. Das getrocknete Kraut riecht unangenehm, der Geschmack salzig-bitter und scharf" (PrentNER 2004). Der Geschmack der rübenartig verdickten Wurzel wird als fettig beschrieben, selbst das bloße Ausgraben führe dazu, dass Menschen betäubt und in Unbesonnenheit versetzt würden (Schmidt 1840). Es wird weiter berichtet, 


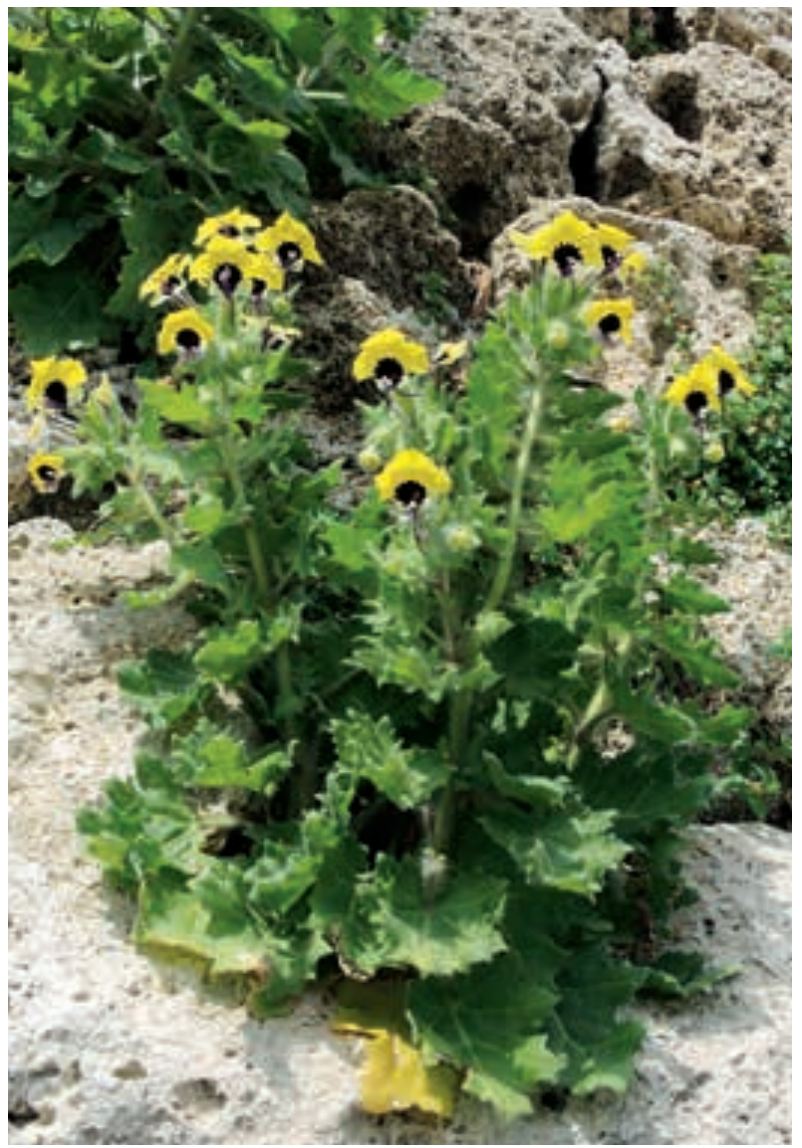

Abb. 9: Hyoscyamus aureus.

dass Verwechslungen mit Zichorienwurzeln zum irrtümlichen Verzehr führen könnten, so dass eine deutliche räumliche Trennung beider Arten im Nutzgarten von Vorteil wäre.

Eine besondere Beziehung hat Hyoscyamus niger schlussendlich auch zum Nationalgetränk der Deutschen. Im Mittelalter wurde dem Bier häufig Bilsenkraut zugesetzt, um die rauschhafte Wirkung zu steigern. Dass die Deutschen auch damals schon wenig Spaß verstanden, wenn es um ihr Lieblingsgetränk ging, beweist der drastische Kommentar in Tabernaemontanus' New Kreuterbuch von 1588 , in dem es heißt, dass diejenigen, die mutwillig das Bier mit Bilsenkraut versetzten, als abgesagte Feinde des menschlichen Geschlechts, als Dieb und Mörder an Leib und Leben zu bestrafen seien. Auch der Name der berühmtesten Bierbrauerstadt, das tschechische Pilsen, wurde wiederholt mit dem Bilsenkraut in Verbindung gebracht, das man dort mutmaßlich in großem Stile angebaut und dem Bier zugesetzt habe (Düll \& Kutzelnigg 2016).

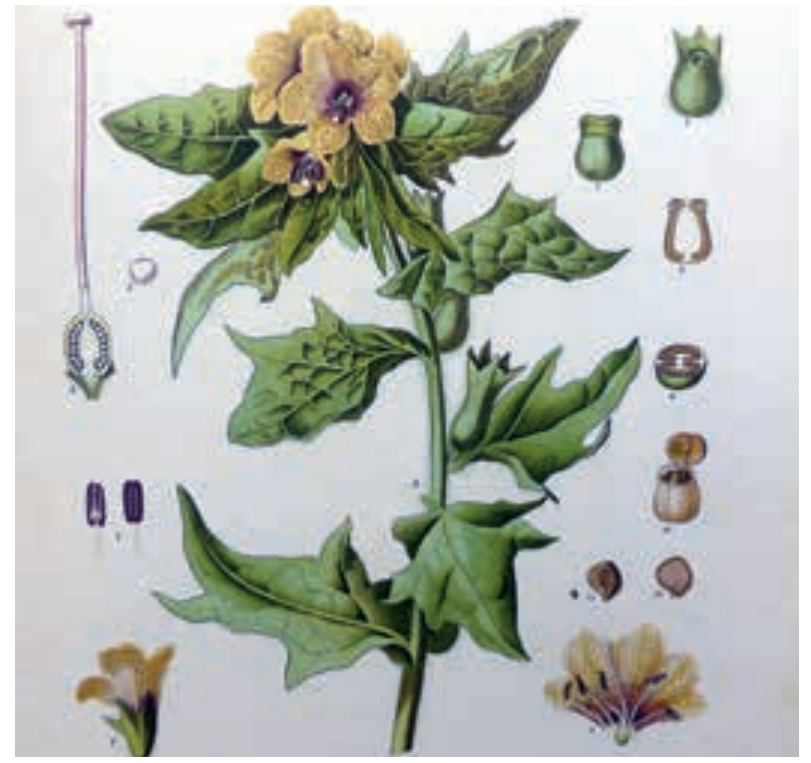

Abb. 10: Hyoscyamus niger, eine wichtige Heilpflanze, dargestellt in KöHLER's Atlas der Medizinalpflanzen (1889).

\section{Weitere Arten und Varietäten}

In Deutschland treten neben der Varietät $H$. niger var. niger nach SCHULTE (1985) noch zwei weitere Varietäten auf, var. pallidus sowie var. agrestis, wobei letztere oft als synonym zur Typusvarietät aufgefasst wird und wohl nur eine Standortmodifikation darstellt (Hegi 1975). Der Varietät var. pallidus fehlt der dunkel gefärbte Schlund, und die Netzaderung ist anders als bei var. niger nur schwach gelblich und nicht violett gefärbt.

Zur Gattung der Bilsenkräuter (Hyoscyamus) gehören 14 Arten, ihr Verbreitungsgebiet erstreckt sich über Europa, Nordafrika und Asien. In Europa selbst kommen neben Hyoscyamus niger noch die zwei von DioskURIDEs erwähnten Arten $H$. albus und $H$. aureus vor. Sie sind beide im Mittelmeergebiet heimisch und wachsen an ruderalen Standorten, nicht selten an alten Mauern und Ruinen. Beide lassen sich von $H$. niger u. a. durch die durchgehend gestielten Blätter, anders gefärbte Blütenkronen sowie schlanker geformte Früchte unterscheiden. In Tab. 1 sind Angaben aus Tutin et al. 1972 und der verschiedenen zitierten Autoren zusammengefügt worden, ergänzt um eigene Beobachtungen auf der Basis von Bildmaterial. Nicht für alle Merkmale konnten genaue Angaben in der Literatur gefunden werden, so dass der Vergleich an dieser Stelle nicht vollständig ist. 
Tab. 1: Bestimmungsmerkmale der Arten Hyoscyamus niger, H. albus und H. aureus

\begin{tabular}{|c|c|c|c|c|c|}
\hline Merkmal & $\begin{array}{l}\text { H. niger } \\
\text { var. niger }\end{array}$ & $\begin{array}{l}\text { H. niger } \\
\text { var. agrestis }\end{array}$ & $\begin{array}{l}\text { H. niger } \\
\text { var. pallidus }\end{array}$ & H. albus & H. aureus \\
\hline Lebensform & $\begin{array}{l}\text { meist } \\
\text { zweijährig }\end{array}$ & einjährig & zweijährig & zweijährig & $\begin{array}{l}\text { ein- bis } \\
\text { mehrjährig }\end{array}$ \\
\hline Größe & $\begin{array}{l}20-80(-100) \\
\mathrm{cm}\end{array}$ & $-30 \mathrm{~cm}$ & $-80 \mathrm{~cm}$ & $-40 \mathrm{~cm}$ & $-50 \mathrm{~cm}$ \\
\hline Stängel & aufrecht & $\begin{array}{l}\text { aufrecht, meist } \\
\text { unverzweigt }\end{array}$ & aufrecht & $\begin{array}{l}\text { aufrecht bis } \\
\text { niederliegend }\end{array}$ & aufrecht \\
\hline Blatt & $\begin{array}{l}\text { untere Blätter } \\
\text { gestielt, obere } \\
\text { sitzend, } \\
\text { stängel- } \\
\text { umfassend }\end{array}$ & $\begin{array}{l}\text { untere Blätter } \\
\text { gestielt, obere } \\
\text { sitzend, } \\
\text { stängel- } \\
\text { umfassend }\end{array}$ & $\begin{array}{l}\text { untere Blätter } \\
\text { gestielt, obere } \\
\text { sitzend, } \\
\text { stängel- } \\
\text { umfassend }\end{array}$ & $\begin{array}{l}\text { alle Blätter } \\
\text { gestielt }\end{array}$ & $\begin{array}{l}\text { alle Blätter } \\
\text { gestielt }\end{array}$ \\
\hline Blüte & $\begin{array}{l}\text { fast sit- } \\
\text { zend; Krone } \\
\text { matt-gelb, } \\
\text { mit violetter } \\
\text { Netzaderung, } \\
\text { Schlund violett } \\
\text { Filament } \\
\text { weiß-gelb } \\
\text { Antheren } \\
\text { violett } \\
\text { Griffel violett } \\
\text { Narbe goldgelb }\end{array}$ & $\begin{array}{l}\text { ähnlich } H \text {. } \\
\text { niger var. niger }\end{array}$ & $\begin{array}{l}\text { fast sitzend, } \\
\text { Krone gelb, } \\
\text { Blütenschlund } \\
\text { gelb, Aderung } \\
\text { nur schwach } \\
\text { durch- } \\
\text { scheinend } \\
\text { gelblich } \\
\text { Antheren } \\
\text { weiß-gelb }\end{array}$ & $\begin{array}{l}\text { sitzend, mit } \\
\text { Ausnahme } \\
\text { der untersten; } \\
\text { Krone blass- } \\
\text { gelb-weiß- } \\
\text { lich, Aderung } \\
\text { nur schwach } \\
\text { durch- } \\
\text { scheinend gelb- } \\
\text { lich, Schlund } \\
\text { violett; } \\
\text { Filament und } \\
\text { Griffel violett, } \\
\text { Narbe blass- } \\
\text { violett, nur } \\
\text { wenig aus } \\
\text { der Krone } \\
\text { herausragend; } \\
\text { Antheren } \\
\text { weiß-gelb }\end{array}$ & $\begin{array}{l}\text { alle Blü- } \\
\text { ten gestielt; } \\
\text { Krone gold- } \\
\text { gelb, Schlund } \\
\text { dunkel-schwarz- } \\
\text { violett, } \\
\text { Filament und } \\
\text { Griffel hell- } \\
\text { rosa bis violett, } \\
\text { Antheren grau- } \\
\text { weiß; weit aus } \\
\text { der Kronröhre } \\
\text { herausragend }\end{array}$ \\
\hline Frucht & $\begin{array}{l}\text { breit glockig, } \\
\text { mittig ein- } \\
\text { geschnürt } \\
\text { mit spitzen } \\
\text { Zähnen, } \\
\text { Basis bauchig } \\
\text { aufgewölbt }\end{array}$ & & & $\begin{array}{l}\text { schlank } \\
\text { glockig, Basis } \\
\text { kaum bauchig } \\
\text { gewölbt }\end{array}$ & $\begin{array}{l}\text { langgezogen } \\
\text { glockig, Basis } \\
\text { kaum bauchig } \\
\text { gewölbt }\end{array}$ \\
\hline
\end{tabular}




\section{Dank}

Ich bedanke mich herzlich bei CoRinne BuCH (Mülheim/Ruhr), Dr. Veit M. Dörken (Konstanz), Annette Höggemeier (Bochum), Harald Geier (Niederkassel), Dr. Armin Jagel (Bochum), Detlef Mährmann (CastropRauxel) und Dario Wolbeck (Attendorn) für die Bereitstellung von Fotos.

\section{Literatur}

Aichele, D. \& Schwegler, H.-W. 2000: Die Blütenpflanzen Mitteleuropas, Bd. 4. - Stuttgart.

Beckhaus, K. 1893: Flora von Westfalen. - Münster (Nachdruck 1993).

Brandes, D. 1977: Über Onopordum acanthium-Gesellschaften in Mitteleuropa. - Doc. Phytosoc. 1: 23-31.

Düll, R. \& Kutzelnigg, H. 2016: Taschenlexikon der Pflanzen Deutschlands und angrenzender Länder, 8. Aufl. Wiebelsheim.

Duve, K. \& Völker, T. 2002: Lexikon berühmter Pflanzen. - München.

FukAREK, F. 2000: Urania-Pflanzenreich, Bd. 2. - Berlin.

Genaust, H. 2005: Etymologisches Wörterbuch der botanischen Pflanzennamen. - Hamburg.

Gorissen, I. 2015: Flora der Region Bonn. - Decheniana, Beih. 40.

Haerkötter, G. \& Haerkötter, M. 1987: Hexenfurz und Teufelsdreck. Liebes-, Heil- und Giftkräuter: Hexereien, Rezepte und Geschichten. - Frankfurt/Main.

Hegi, G. 1975: Illustrierte Flora von Mitteleuropa, Bd. 5(4), 2. Aufl. - Berlin.

Haeupler, H., Jagel, A. \& Schumacher, W. 2003: Verbreitungsatlas der Farn- und Blütenpflanzen Nordrhein-Westfalens. - Recklinghausen.

Höppner, H. \& Preus, H. 1926: Flora des Westfälisch-Rheinischen-Industriegebiets unter Einschluss der Rheinischen Bucht. - Duisburg. (Nachdruck 1971).

Kluge, F. \& Seebold, E. 2002: Etymologisches Wörterbuch der deutschen Sprache, 24. Aufl. - Berlin.

Laven, L. \& Thyssen, T. 1959: Flora des Köln-Bonner Wandergebietes. - Decheniana 112(1): 1-179.
Lienenbecker, H. \& RaAbe, U. 1993: Die Dorfflora Westfalens. - Ilex-Bücher 3 (Bielefeld).

Marzell, H. 1972: Wörterbuch der deutschen Pflanzennamen. - Leipzig.

Möвıus, M. 1931. Die Bestäubung von Hyoscyamus aureus. Ber. Deutsch. Bot. Ges. 49: 359-363.

Philippi, G. 1996: Solanaceae. In: Sebald, O., Seybold, S., Philippi, G. \& Wörz, A.: Die Farn- und Blütenpflanzen Baden-Württembergs, Bd. 5. - Stuttgart.

Ротт, R. 1995: Die Pflanzengesellschaften Deutschlands, 2. Aufl. - Stuttgart.

Preising, E., Vahle, H.-C., Brandes, D., Hofmeister, H., Tüxen, J. \& Weber, H. E. 1993: Die Pflanzengesellschaften Niedersachsens - Bestandsentwicklung, Gefährdung und Schutzprobleme. Ruderale Staudenfluren und Saumgesellschaften. - Hannover.

Prentner, A. 2004: Bewusstseinsverändernde Pflanzen von A-Z. - Wien, New York.

Raabe, U., Büscher, D., Fasel, P., Foerster, E., Götte, R., Haeupler, H., Jagel, A., Kaplan, K., Keil, P., Kulbrock, P., Loos, G. H., Neikes, N., Schumacher, W., Sumser, H. \& Vanberg, C. 2011: Rote Liste und Artenverzeichnis der Farn- und Blütenpflanzen - Pteridophyta et Spermatophyta - in Nordrhein-Westfalen. - Rcklinghausen.

Roth, L., Daunderer, M. \& Kormann, K. 2012: Giftpflanzen. - Pflanzengifte, 6. Aufl. - Hamburg.

Schläppi, M, 2011: Genetic and physiological analysis of biennialism in Hyoscyamus niger. - Plant Biology, 13: 534 540 (http://onlinelibrary.wiley.com/doi/10.1111/j.14388677.2010.00382.x/full).

Schmidt, J. A. F. 1840: Vollständiges Giftbuch. - Weimar.

Schulte, W. 1985: Hyoscyamus niger var. pallidus Waldst. \& Kiт. (Gelbblühende Varietät des Schwarzen Bilsenkrautes) auf ehemaliger Industriebrache in Hamm (Westf.). - Göttinger Florist. Rundbr. 19: 39-42.

Tutin, T. G., Heywood, V. H., Burges, N. A., Moore, D. M., Valentine, D. H., Walters, S. M. \& Webb, D. A. 1972: Flora Europaea. - Cambridge.

\section{Internetseite}

Pharmawiki 2017: Dioskurides. Materia medica. Buch IV, 69 Bilsenkraut - http://www.pharmawiki.ch/materiamedica/ index.php?page=Home 